\title{
Modify adaptive combined synchronization of fractional or- der chaotic systems with fully unknown parameters
}

\author{
A. Othman Almatroud ${ }^{a}, *$, O. Ababneh ${ }^{b}$, M. Mossa Al-sawalha ${ }^{a}$ \\ a Mathematics Department, Faculty of Science, University of Ha'il, Kingdom of Saudi Arabia. \\ ${ }^{b}$ School of Mathematics, Zarqa University, Zarqa, Jordan.
}

\begin{abstract}
This article presents a modify adaptive combined synchronization for a class of different unknown fractional order chaotic systems. A combination of different states of the drive systems asymptotically synchronizes with the desired states of the response system. Hence, increases the complexity of the communication channel in secrete communications. The Lyapunov stability theory proves the asymptotic stability of the error system at the origin. The design of a suitable adaptive controller assures the target synchronization. This work provides parameters update laws that estimate the true values of unknown parameters. This paper also presents two numerical examples of unknown different fractional order chaotic systems and simulation results that validate the efficiency and performance of the proposed adaptive combined synchronization strategy. The presented adaptive combined synchronization strategy can be applied to multiple synchronization strategies. The paper suggests some future problems related to this work.
\end{abstract}

Keywords: Chaos, combined synchronization, adaptive control, unknown parameters, fractional order.

2020 MSC: 93D99, 93C40.

(C)2020 All rights reserved.

\section{Introduction}

The study of complex dynamical systems has a long history as several natural phenomena exhibit dynamics which are many a times quite complex to study. This inherent complexity of systems presents challenges in the investigation of their characteristics and it had been the case up until early 1980s due to the limitation of computational power. However, this barrier has slowly been overcome with the fast speed computers which made it possible the exploration of territories which were once considered highly infeasible if not impossible.

Synchronization of chaotic systems is one area which has received great attention for over several decades now. Extensive studies have been conducted on the synchronization of chaotic systems of integer order but a fast emerging trend is the attention towards the more general order which includes the fractional order systems. Although the applications of integer order systems are more prevalent, innumerable

\footnotetext{
${ }^{*}$ Corresponding author

Email address: othman_almatroud@yahoo.com (A. Othman Almatroud)
}

doi: $10.22436 /$ jmcs.021.02.01

Received: 2019-11-09 Revised: 2020-01-15 Accepted: 2020-02-12 
and steadily growing, however, the fractional order chaotic systems is picking the pace now as they are more effective in modelling natural complex systems than their counterpart integer order systems. The science of fractional order chaos has paved into several academic disciplines such as engineering, sciences such as biology, chemistry, physics as well as in the areas of nonlinear science and it is considered as an interdisciplinary field now $[19,27,39,41]$. The use of fractional order chaotic dynamics is prevalent in the control and synchronization of fractional order chaotic systems. The aforementioned topics present a fine blending of the mathematics of the fractional order chaos with that of the numerous applications. At present, synchronization of fractional order chaotic systems becomes a challenging and interesting problem due to its potential applications in secure communication and control processing, chemical reactions, biological systems, etc. The concept of the fractional order dynamical systems has a theoretical basis in fractional calculus which is a generalization of classical calculus. It may sound relatively a new frontier nevertheless its traces are found even in the works of the founding fathers of ordinary calculus such as Leibniz and other the contemporary mathematicians [15, 31].

After the pioneering work Pecora and Carrol [26], considerable attention has been paid to the research of synchronizing fractional order chaotic systems. For the last three decades, fractional order chaos synchronization has been remained an interesting subject due to its potential applications in secure communications, chemical reactions, salt-water oscillators, electronic circuits and neural networks, etc. A number of effective control techniques have been developed to accomplish the fractional order chaotic synchronization, such as the active and backstepping control techniques, nonlinear control strategy, adaptive control approach, sliding mode control method, and intermittent pinning control technique, $[1-5,9-12,14,16,17,21,28-30,34,35,38,40,42,43,45]$.

In the recent decade, the one-to-one system synchronization mode has been shifted to new types of synchronization strategies where more than two chaotic systems are involved in drive-response system arrangement. In this line, based on the chaos synchronization theory, different synchronization control schemes have been developed. These include, compound synchronization [37], dual combination synchronization [33], multi switching combination synchronization [7, 32], dual combination-combination multi switching synchronization [6] and dual synchronization [22-25]. These approaches involve the same number of fractional order chaotic systems in the drive-response system synchronization mode. Such type of synchronization approaches are meaningful in secure communication systems, for instance, the transmitted signals can be divided in to several parts, loaded in different chaotic signals of the transmitter system and the original message signal can be recovered in the receiver system with enough security. Hence, increases the security of the information signal in communication systems. Until, the research on fractional order chaos synchronization is still open and challenging.

To the best of authors' knowledge based on the literature survey, there is no result in the relevant literature dealing with the adaptive compound synchronization among four unknown fractional order chaotic systems containing three unknown fractional order chaotic systems in the drive system and one unknown fractional order chaotic system in the response system. The aforesaid discussion motivates us to study the adaptive combined synchronization among four different fractional order chaotic systems with unknown parameters. These systems have different parameters values. Based on the Lyapunov stability theory [20] and using the adaptive control strategy [1], sufficient conditions are derived analytically that established the asymptotic stability of the error dynamical system at the origin. Suitable adaptive laws are derived to estimate the exact value of the unknown parameters.

Two numerical examples are presented. In the first example, a combination of two different unknown fractional order chaotic Lü, Liu systems are considered as the drive system and the unknown Chen fractional order chaotic system is considered as the response system. In the second example, a combination of the unknown fractional order chaotic Lü, Liu and Genesio-Tesi systems are considered as the drive system and the unknown fractional order Chen chaotic system is considered as the response system. Numerical simulation results are furnished to validate the theoretical findings. Since the fractional order chaotic systems under study have different parameter values and these parameters are assumed unknown. Hence, the proposed approach increases the complexity level of the drive (transmitter) system, thereby, enriches 
the security of the information signal in secure communication systems. The rest of the paper is organized as follows. Section 2 presents a step by step methodology for the proposed adaptive combined synchronization controller design of fractional order chaotic systems with unknown parameters. In Section 3, description of the fractional order chaotic Lü, Liu, Genesio-Tesi and Chen systems are given, followed by two numerical examples for the adaptive combined synchronization with simulations in Section 4 . This paper concludes in Section 5 with some future problems related to this work.

\section{Preliminaries of fractional-order calculus}

The concept of an integer-order integro-differential operator can be extending by the fractional-order integro-differential operator using a generalizable formulation, that is

$$
{ }_{a} D_{t}^{p}= \begin{cases}\frac{d^{p}}{d t^{p}}, & p>0 \\ 1, & p=0 \\ \int_{a}^{t}(d \tau)^{-p}, & p<0\end{cases}
$$

where $p$ is the fractional order which could be a complex number, and $a, t$ symbolize the limits of the operation. There are many definitions of the fractional integral and derivative which have been used in the recent literature, precisely, the following three definitions (Grünwald-Letnikov, Riemann-Liouville, and Caputo). The current study dealing with the Riemann-Liouvile definition ([1,27]), which is given by

$$
{ }_{a} D_{t}^{p} f(t)=\frac{d^{m}}{d t^{m}} J_{t}^{m-}-p_{f}(t), \quad p>0,
$$

where $\mathrm{m}=\lceil\mathrm{p}\rceil, \mathrm{J}$ is the fractional Riemann-Liouville integral and

$$
J_{t}^{\varphi} \phi(t)=\frac{1}{\Gamma(\varphi)} \int_{0}^{t} \frac{\phi(v)}{(t-v)^{1-\varphi}} d v
$$

with $0<\varphi \leqslant 1$ and $\Gamma$ (.) is a gamma function. For $r>n \geqslant 0, p$ and $q$ are integers such that $0 \leqslant p-1 \leqslant$ $\mathrm{r}<\mathrm{p}$, and $0 \leqslant \mathrm{q}-1 \leqslant \mathrm{n}<\mathrm{q}$. Then,

$$
{ }_{a} D_{t}^{r}\left({ }_{a} D_{t}^{-} m_{f}(t)\right)={ }_{a} D_{t}^{r-m} f(t) .
$$

For $r, m \geqslant 0$, there exist integers $p$ and $q$ such that $0 \leqslant p-1 \leqslant r<p$, and $0 \leqslant q-1 \leqslant m<q$. Then,

$$
{ }_{a} D_{t}^{r}\left({ }_{a} D_{t}^{m} f(t)\right)={ }_{a} D_{t}^{r+m} f(t)-\sum_{j=1}^{m}\left[{ }_{a} D_{t}^{m-j} f(t)\right]_{t=a} \frac{(t-a)^{-r-j}}{\Gamma(1-r-j)} .
$$

Suppose also that $f(t)$ has a continuous $n$th derivative in $[0, t](n \in N, t>0)$ and let $r, m>0$. Then, there exists some $k \in N$ with $k \leqslant n$ and $r, r+m \in[k-1, k]$ such that

$$
{ }_{a} D_{t a}^{r} D_{t}^{m} f(t)={ }_{a} D_{t}^{r+m} f(t) .
$$

\section{Adaptive combined synchronization controller design}

Let us consider the following chaotic systems:

$$
\begin{aligned}
D_{\mathrm{t}}^{s} x_{1} & =g_{1}\left(x_{1}\right)+G_{1}\left(x_{1}\right) \varphi_{1}, \\
D_{t}^{s} x_{2} & =g_{2}\left(x_{2}\right)+g_{1}\left(x_{2}\right) \varphi_{2}, \\
D_{t}^{s} x_{3} & =g_{3}\left(x_{3}\right)+g_{3}\left(x_{3}\right) \varphi_{3}, \\
& \vdots \\
D_{t}^{s} x_{k} & =g_{k}\left(x_{k}\right)+g_{k}\left(x_{k}\right) \varphi_{k},
\end{aligned}
$$


where $x_{i} \in \Omega_{x_{i}} \subset \Re^{p}$ is the state vector. Let $\Omega=\bigcap_{i=1}^{k} \Omega_{x_{i}} \neq \phi$ such that $\sum_{i=1}^{k} x_{i}$ is defined on $\Omega, \varphi_{i} \in \Re^{p}$ is the unknown parameter vector of the system $x_{i}, g_{i}\left(x_{i}\right)$ is a continuous vector function, $G_{i}\left(x_{i}\right)$ is a matrix function. On the other hand, the controlled response system is assumed by

$$
D_{t}^{s} y=h(y)+H(y) \vartheta+u,
$$

where $y \in \Omega_{y} \subset \mathfrak{R}^{p}$ is the state vector, $\vartheta \in \mathfrak{R}^{p}$ is the unknown parameter vector of the system, $h(y)$ is a continuous vector function, $H(y)$ is a matrix function, $u \in \mathfrak{R}^{p}$ is a controller. Let $E(t)=y(t)-\left(x_{1}(t)+\right.$ $\left.x_{2}(t)+\cdots+x_{k}(t)\right)$ be the error vector. Our aim is to find a suitable control function $u$ which can able to achieve the combined synchronization such that,

$$
\lim _{t \rightarrow \infty}|E|=\lim _{t \rightarrow \infty}\left|y\left(t, y_{0}\right)-\left(x_{1}\left(t, x_{10}\right)+x_{2}\left(t, x_{20}\right)+\cdots+x_{k}\left(t, x_{k 0}\right)\right)\right|=0
$$

The following theorem shows that the systems (3.1) and (3.2) can be effectively combined synchronized.

Theorem 3.1. If the adaptive combined control laws are chosen as follows:

$$
\begin{aligned}
u= & g_{1}\left(x_{1}\right)+G_{1}\left(x_{1}\right) \varphi_{1}+g_{2}\left(x_{2}\right)+G_{2}\left(x_{2}\right) \varphi_{2}+\cdots \\
& +g_{k}\left(x_{k}\right)+G_{k}\left(x_{k}\right) \varphi_{k}-h(y)-H(y) \vartheta+D_{t}^{s-1}\left[G_{1}\left(x_{1}\right) \hat{\varphi}_{1}+G_{2}\left(x_{2}\right) \hat{\varphi}_{2}+\cdots\right. \\
& \left.+G_{k}\left(x_{k}\right) \hat{\varphi}_{k}-H(y) \hat{\vartheta}-\left(D_{t}^{s-1} E(t)\right) \frac{(t)^{-(s-1)-1}}{\Gamma(-(s-1))}-E\right]
\end{aligned}
$$

and the update rule of the unknown parameters are taken as

$$
\begin{aligned}
\dot{\hat{\varphi}}_{1} & =-\left[G_{1}\left(x_{1}\right)\right]^{\top} e, \\
\dot{\hat{\varphi}}_{2} & =-\left[G_{2}\left(x_{2}\right)\right]^{\top} e, \\
\vdots & \\
\dot{\hat{\varphi}}_{k} & =-\left[G_{k}\left(x_{k}\right)\right]^{\top} e, \\
\dot{\hat{\vartheta}} & =[H(y)]^{\top} e,
\end{aligned}
$$

where $s \in[0,1]$ is the fractional order and $\hat{\varphi}_{1}, \hat{\varphi}_{2}, \ldots, \hat{\varphi}_{k}, \hat{\vartheta}$ are the estimated parameters of $\varphi_{1}, \varphi_{2}, \ldots, \varphi_{k}$ and $\vartheta$, respectively, then the drive systems (3.1) will achieve adaptive combined synchronization with the response systems (3.2).

Proof. The error dynamical system between drive system (3.1) and response system (3.2) is described by

$$
\begin{aligned}
D_{t}^{s} E(t)= & h(y)+H(y) \vartheta-g_{1}\left(x_{1}\right)-G_{1}\left(x_{1}\right) \varphi_{1}-g_{2}\left(x_{2}\right) \\
& -G_{2}\left(x_{2}\right) \varphi_{2}-\cdots-g_{k}\left(x_{k}\right)-G_{k}\left(x_{k}\right) \varphi_{k}+u,
\end{aligned}
$$

where $E=y-\left(x_{1}+x_{2}+\cdots+x_{k}\right)$. Substituting (3.3) into (3.5) results in the following

$$
\begin{aligned}
D_{t}^{s} E(t)= & D_{t}^{s-1}\left[F_{1}\left(x_{1}\right) \tilde{\varphi}_{1}+F_{2}\left(x_{2}\right) \tilde{\varphi}_{2}+\cdots+F_{k}\left(x_{k}\right) \tilde{\varphi}_{k}-G(y) \tilde{\vartheta}\right. \\
& \left.-\left(D_{t}^{s-1} E(t)\right) \frac{(t)^{-(s-1)-1}}{\Gamma(-(s-1))}-E\right],
\end{aligned}
$$


where, $\tilde{\varphi}_{1}=\hat{\varphi}_{1}-\varphi_{1}, \tilde{\varphi}_{2}=\hat{\varphi}_{2}-\varphi_{2}, \ldots, \tilde{\varphi}_{k}=\hat{\varphi}_{k}-\varphi_{k}, \tilde{\vartheta}=\hat{\vartheta}-\vartheta$. Construct a Lyapunov function candidate as

$$
V=\frac{1}{2}\left[E^{\top} E+\tilde{\varphi}_{1}^{\top} \tilde{\varphi}_{1}+\tilde{\varphi}_{2}^{\top} \tilde{\varphi}_{2}+\cdots+\tilde{\varphi}_{k}^{\top} \tilde{\varphi}_{k}+\tilde{\vartheta}^{\top} \tilde{\vartheta}\right]
$$

The derivative of $V$ along the solutions of the error dynamical system (3.5) is

$$
\dot{V}=\mathrm{E}^{\mathrm{T}} \dot{\mathrm{E}}+\dot{\tilde{\varphi}}_{1}^{\top} \tilde{\varphi}_{1}+\dot{\tilde{\varphi}}_{2}^{\top} \tilde{\varphi}_{2}+\cdots+\dot{\tilde{\varphi}}_{k} \tilde{\varphi}_{k}+\dot{\tilde{\vartheta}}^{\top} \tilde{\vartheta}
$$

Thus, using (2.1), we have

$$
\begin{aligned}
\dot{V}= & E^{\top}\left[D_{t}^{s-1}\left(D_{t}^{s} E(t)\right)+\left(D_{t}^{s-1} E(t)\right) \frac{(t)^{-(s-1)-1}}{\Gamma(-(s-1))}\right] \\
& +\dot{\tilde{\varphi}}_{1}^{\top} \tilde{\varphi}_{1}+\dot{\varphi}_{2}^{\top} \tilde{\varphi}_{2}+\cdots+\dot{\tilde{\varphi}}_{k} \tilde{\varphi}_{k}+\dot{\tilde{\vartheta}}^{\top} \tilde{\vartheta} .
\end{aligned}
$$

Hence, from (3.6), we obtain,

$$
\begin{aligned}
\dot{V}= & E^{\top}\left[D _ { t } ^ { s - 1 } \left(D _ { t } ^ { s - 1 } \left[G_{1}\left(x_{1}\right) \tilde{\varphi}_{1}+G_{2}\left(x_{2}\right) \tilde{\varphi}_{2}\right.\right.\right. \\
& \left.+\cdots+G_{k}\left(x_{k}\right) \tilde{\varphi}_{k}-H(y) \tilde{\vartheta}-\left(D_{t}^{s-1} E(t)\right) \frac{(t)^{-(s-1)-1}}{\Gamma(-(s-1))}-E\right] \\
& \left.+\left(D_{t}^{s-1} E(t)\right) \frac{(t)^{-(s-1)-1}}{\Gamma(-(s-1))}\right]+\dot{\tilde{\varphi}}_{1}^{\top} \tilde{\varphi}_{1}+\dot{\tilde{\varphi}}_{2}^{\top} \tilde{\varphi}_{2}+\cdots+\dot{\tilde{\varphi}}_{k} \tilde{\varphi}_{k}+\dot{\tilde{\vartheta}}^{\top} \tilde{\vartheta} .
\end{aligned}
$$

Using (2.2) and (3.4), (3.7) reduces to

$$
\begin{aligned}
\dot{V}= & E^{\top}\left[G_{1}\left(x_{1}\right) \tilde{\varphi}_{1}+G_{2}\left(x_{2}\right) \tilde{\varphi}_{2}+\cdots+G_{k}\left(x_{k}\right) \tilde{\varphi}_{k}-H(y) \tilde{\vartheta}-\left(D_{t}^{s-1} E(t)\right) \frac{(t)^{-(s-1)-1}}{\Gamma(-(s-1))}-E\right. \\
& \left.+\left(D_{t}^{s-1} E(t)\right) \frac{(t)^{-(s-1)-1}}{\Gamma(-(s-1))}\right]-E^{\top}\left[G 1\left(x_{1}\right) \tilde{\varphi}_{1}+G_{2}\left(x_{2}\right) \tilde{\varphi}_{2}+\cdots+G_{k}\left(x_{k}\right) \tilde{\varphi}_{k}-H(y) \tilde{\vartheta}\right] \\
= & -E^{\top} E \leqslant 0 .
\end{aligned}
$$

Based on Lyapunov theory [20], the combined synchronization is achieved between the drive system (3.1) and the response system (3.2) under the choice of the controller $u$ (3.3) and parameters update law (3.4) is achieved.

\section{Adaptive combined synchronization of Lü, Liu and Chen systems}

In this section, we shall extend the direct application of the proposed above method to study the combined synchronization of the fraction order Lü [8], Liu [18] and Chen systems. Here we have assumed that the fraction order Lü, Liu systems as a drive systems and fraction order Chen [44] system assumed as a response system as follows:

$$
\begin{array}{lll}
D_{t}^{s_{1}} x_{1}=a_{1}\left(y_{1}-x_{1}\right), & D_{t}^{s_{2}} y_{1}=-x_{1} z_{1}+c_{1} y_{1}, & D_{t}^{s_{3}} z_{1}=x_{1} y_{1}-b_{1} z_{1}, \\
D_{t}^{s_{1}} x_{2}=a_{2}\left(y_{2}-x_{2}\right), & D_{t}^{s_{2}} y_{2}=b_{2} x_{2}-x_{2} z_{2}, & D_{t}^{s_{3}} z_{2}=-c_{2} z_{2}+d_{2} x_{2}^{2} \\
D_{t}^{s_{1}} x_{3}=a_{3}\left(y_{3}-x_{3}\right)+u_{1}, & D_{t}^{s_{2}} y_{3}=\left(c_{3}-a_{3}\right) x_{3}-x_{3} z_{3}+c_{3} y_{3}+u_{2}, & D_{t}^{s_{3}} z_{2}=x_{3} y_{3}-b_{3} z_{3}+u_{3},
\end{array}
$$

where $\mathrm{U}=\left(\mathfrak{u}_{1}, \mathfrak{u}_{2}, \mathfrak{u}_{3}\right)^{\top}$ denote to the controller function. In the following, an effective adaptive controller function is constructed to achieve the combined synchronization between Lü, Liu systems and Chen 
system with fully unknown parameters. By subtracting (4.1)-(4.2) from (4.3) the following error system is obtained:

$$
\begin{aligned}
& D_{t}^{s_{1}} e_{1}(t)=a_{3}\left(y_{3}-x_{3}\right)-a_{2}\left(y_{2}-x_{2}\right)-a_{1}\left(y_{1}-x_{1}\right)+u_{1}, \\
& D_{t}^{s_{2}} e_{2}(t)=\left(c_{3}-a_{3}\right) x_{3}-x_{3} z_{3}+c_{3} y_{3}-b_{2} x_{2}+x_{2} z_{2}+x_{1} z_{1}-c_{1} y_{1}+u_{2}, \\
& D_{t}^{s_{3}} e_{3}(t)=x_{3} y_{3}-b_{3} z_{3}+c_{2} z_{2}-d_{2} x_{2}^{2}-x_{1} y_{1}+b_{1} z_{1}+u_{3}
\end{aligned}
$$

where $e_{1}=x_{3}-x_{2}-x_{1}, e_{2}=y_{3}-y_{2}-y_{1}$ and $e_{3}=z_{3}-z_{2}-z_{1}$. Our objective is to design the controller $\mathrm{U}$ with a parameter update law in order to achieve the globally and asymptotically combined synchronization between (4.1)-(4.2) and (4.3).

In order to show that systems (4.1)-(4.2) and system (4.3) are combined synchronized, the following Theorem is construct.

Theorem 4.1. The asymptotically combined synchronization of the systems (4.1)-(4.2) and the system (4.3) will achieve for any distinct initial condition with following modify adaptive controller

$$
\begin{aligned}
u_{1}= & -a_{3}\left(y_{3}-x_{3}\right)+a_{2}\left(y_{2}-x_{2}\right)+a_{1}\left(y_{1}-x_{1}\right)+D_{t}^{s_{1}-1}\left[-\hat{a}_{3}\left(y_{3}-x_{3}\right)+\hat{a}_{2}\left(y_{2}-x_{2}\right)\right. \\
& \left.+\hat{a}_{1}\left(y_{1}-x_{1}\right)-\left(D_{t}^{s_{1}-1} e_{1}(t)\right) \frac{(t)^{-\left(s_{1}-1\right)-1}}{\Gamma\left(-\left(s_{1}-1\right)\right)}-e_{1}\right], \\
u_{2}= & -\left(c_{3}-a_{3}\right) x_{3}+x_{3} z_{3}-c_{3} y_{3}+b_{2} x_{2}-x_{2} z_{2}-x_{1} z_{1}+c_{1} y_{1}+D_{t}^{s_{2}-1}\left[-\left(\hat{c}_{3}-\hat{a}_{3}\right) x_{3}-\hat{c}_{3} y_{3}\right. \\
& \left.+\hat{b}_{2} x_{2}+\hat{c}_{1} y_{1}-\left(D_{t}^{s_{2}-1} e_{2}(t)\right) \frac{(t)^{-\left(s_{2}-1\right)-1}}{\Gamma\left(-\left(s_{2}-1\right)\right)}-e_{2}\right], \\
u_{3}= & -x_{3} y_{3}+b_{3} z_{3}-c_{2} z_{2}+d_{2} x_{2}^{2}+x_{1} y_{1}-b_{1} z_{1}+D_{t}^{s_{3}-1}\left[\hat{b}_{3} z_{3}-\hat{c}_{2} z_{2}+\hat{d}_{2} x_{2}^{2}-b_{1} z_{1}\right. \\
& \left.-\left(D_{t}^{s_{3}-1} e_{3}(t)\right) \frac{(t)^{-\left(s_{3}-1\right)-1}}{\Gamma\left(-\left(s_{3}-1\right)\right)}-e_{3}\right],
\end{aligned}
$$

and the update rule of the unknown parameters are taken as

$$
\begin{aligned}
& \dot{\tilde{\mathrm{a}}}_{1}=-\left(\mathrm{y}_{1}-\mathrm{x}_{1}\right) \mathrm{e}_{1}, \quad \dot{\tilde{\mathrm{b}}}_{1}=z_{1} \mathrm{e}_{3}, \quad \dot{\tilde{\mathrm{c}}}_{1}=-\mathrm{y}_{1} \mathrm{e}_{2}, \quad \dot{\tilde{\mathrm{a}}}_{2}=-\left(\mathrm{y}_{2}-\mathrm{x}_{2}\right) \mathrm{e}_{1}, \quad \dot{\tilde{\mathrm{b}}}_{2}=-\mathrm{x}_{2} \mathrm{e}_{2}, \\
& \dot{\tilde{c}}_{2}=z_{2} e_{3}, \quad \dot{\tilde{d}}_{2}=-x_{2}^{2} e_{3}, \quad \dot{\tilde{a}}_{3}=\left(y_{3}-x_{3}\right) e_{1}-x_{3} e_{2}, \quad \dot{\tilde{b}}_{3}=-z_{3} e_{3}, \quad \dot{\tilde{c}}_{3}=\left(x_{3}+y_{3}\right) e_{2},
\end{aligned}
$$

where $\hat{a}_{1}, \hat{b}_{1}, \hat{c}_{1}, \hat{a}_{2}, \hat{b}_{2}, \hat{c}_{2}, \hat{d}_{2}, \hat{a}_{3}, \hat{b}_{3}, \hat{c}_{3}$ are estimates of $\mathrm{a}_{1}, \mathrm{~b}_{1}, \mathrm{c}_{1}, \mathrm{a}_{2}, \mathrm{~b}_{2}, \mathrm{c}_{2}, \mathrm{~d}_{2}, \mathrm{a}_{3}, \mathrm{~b}_{3}, \mathrm{c}_{3}$, respectively.

Proof. By applying the modify adaptive controller (4.5) to (4.4), the following new is error systems obtained:

$$
\begin{aligned}
& D_{t}^{s_{1}} e_{1}(t)=D_{t}^{s_{1}-1}\left[-\tilde{a}_{3}\left(y_{3}-x_{3}\right)+\tilde{a}_{2}\left(y_{2}-x_{2}\right)+\tilde{a}_{1}\left(y_{1}-x_{1}\right)-\left(D_{t}^{s_{1}-1} e_{1}(t)\right) \frac{(t)^{-\left(s_{1}-1\right)-1}}{\Gamma\left(-\left(s_{1}-1\right)\right)}-e_{1}\right], \\
& D_{t}^{s_{2}} e_{2}(t)=D_{t}^{s_{2}-1}\left[-\left(\tilde{c}_{3}-\tilde{a}_{3}\right) x_{3}-\tilde{c}_{3} y_{3}+\tilde{b}_{2} x_{2}+\tilde{c}_{1} y_{1}-\left(D_{t}^{s_{2}-1} e_{2}(t)\right) \frac{(t)^{-\left(s_{2}-1\right)-1}}{\Gamma\left(-\left(s_{2}-1\right)\right)}-e_{2}\right], \\
& D_{t}^{s_{3}} e_{3}(t)=D_{t}^{s_{3}-1}\left[\tilde{b}_{3} z_{3}-\tilde{c}_{2} z_{2}+\tilde{d}_{2} x_{2}^{2}-b_{1} z_{1}-\left(D_{t}^{s_{3}-1} e_{3}(t)\right) \frac{(t)^{-\left(s_{3}-1\right)-1}}{\Gamma\left(-\left(s_{3}-1\right)\right)}-e_{3}\right],
\end{aligned}
$$

where $\tilde{a}_{1}=\hat{a}_{1}-a_{1}, \tilde{b}_{1}=\hat{b}_{1}-b_{1}, \tilde{c}_{1}=\hat{c}_{1}-c_{1}, \tilde{a}_{2}=\hat{a}_{2}-a_{2}, \tilde{b}_{2}=\hat{b}_{2}-b_{2}, \tilde{c}_{2}=\hat{c}_{2}-c_{2}, \tilde{d}_{2}=\hat{d}_{2}-d_{2}, \tilde{a}_{3}=$ $\hat{a}_{3}-a_{3}, \tilde{b}_{3}=\hat{b}_{3}-b_{3}, \tilde{c}_{3}=\hat{c}_{3}-c_{3}$. Construct a Lyapunov function candidate as

$$
V=\frac{1}{2}\left(e^{\top} e+\tilde{a}_{1}^{2}+\tilde{b}_{1}^{2}+\tilde{c}_{1}^{2}+\tilde{a}_{2}^{2}+\tilde{b}_{2}^{2}+\tilde{c}_{2}^{2}+\tilde{\mathrm{d}}_{2}^{2}+\tilde{\mathrm{a}}_{3}^{2}+\tilde{\mathrm{b}}_{3}^{2}+\tilde{\mathrm{c}}_{3}^{2}\right)
$$

Differentiate $\mathrm{V}$ with respect to time the following is obtained:

$$
\dot{\mathrm{V}}=\left(e^{\top} \dot{\mathrm{e}}+\tilde{\mathrm{a}}_{1} \dot{\tilde{\mathrm{a}}}_{1}+\tilde{\mathrm{b}}_{1} \dot{\tilde{\mathrm{b}}}_{1}+\tilde{\mathrm{c}}_{1} \dot{\tilde{\mathrm{c}}}_{1}+\tilde{\mathrm{a}}_{2} \dot{\tilde{\mathrm{a}}}_{2}+\tilde{\mathrm{b}}_{2} \dot{\tilde{\mathrm{b}}}_{2}+\tilde{\mathrm{c}}_{2} \dot{\tilde{\mathrm{c}}}_{2}+\tilde{\mathrm{d}}_{2} \dot{\tilde{\mathrm{d}}}_{2}+\tilde{\mathrm{a}}_{3} \dot{\tilde{\mathrm{a}}}_{3}+\tilde{\mathrm{b}}_{3} \dot{\tilde{\mathrm{b}}}_{3}+\tilde{\mathrm{c}}_{3} \dot{\tilde{\mathrm{c}}}_{3}\right),
$$

using (2.1) in (4.7) yields the following 


$$
\begin{aligned}
& \dot{V}=\left(e_{1}\left[D_{t}^{1-s_{1}}\left(D_{t}^{s_{1}} e_{1}(t)\right)+\left(D_{t}^{s_{1}} e_{1}(t)\right) \frac{(t)^{-\left(s_{1}-1\right)-1}}{\Gamma\left(-\left(s_{1}-1\right)\right)}\right]+e_{2}\left[D_{t}^{1-s_{2}}\left(D_{t}^{s_{2}} e_{2}(t)\right)\right.\right. \\
& \left.+\left(D_{t}^{s_{2}} e_{2}(t)\right) \frac{(t)^{-\left(s_{2}-1\right)-1}}{\Gamma\left(-\left(s_{2}-1\right)\right)}\right]+e_{3}\left[D_{t}^{1-s_{3}}\left(D_{t}^{s_{3}} e_{3}(t)\right)+\left(D_{t}^{s_{3}} e_{3}(t)\right) \frac{(t)^{-\left(s_{3}-1\right)-1}}{\Gamma\left(-\left(s_{3}-1\right)\right)}\right]
\end{aligned}
$$

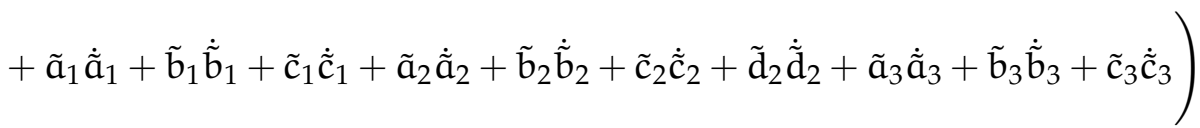

$$
\begin{aligned}
& =\left(e _ { 1 } \left[D _ { t } ^ { 1 - s _ { 1 } } \left(D _ { t } ^ { s _ { 1 } - 1 } \left[-\tilde{a}_{3}\left(y_{3}-x_{3}\right)+\tilde{a}_{2}\left(y_{2}-x_{2}\right)+\tilde{a}_{1}\left(y_{1}-x_{1}\right)\right.\right.\right.\right. \\
& \left.\left.-\left(D_{t}^{s_{1}-1} e_{1}(t)\right) \frac{(t)^{-\left(s_{1}-1\right)-1}}{\Gamma\left(-\left(s_{1}-1\right)\right)}-e_{1}\right]+\left(D_{t}^{s_{1}} e_{1}(t)\right) \frac{(t)^{-\left(s_{1}-1\right)-1}}{\Gamma\left(-\left(s_{1}-1\right)\right)}\right] \\
& +e_{2}\left[D _ { t } ^ { 1 - s _ { 2 } } \left(D _ { t } ^ { s _ { 2 } - 1 } \left[-\left(\tilde{c}_{3}-\tilde{a}_{3}\right) x_{3}-\tilde{c}_{3} y_{3}+\tilde{b}_{2} x_{2}+\tilde{c}_{1} y_{1}\right.\right.\right. \\
& \left.\left.-\left(D_{t}^{s_{2}-1} e_{2}(t)\right) \frac{(t)^{-\left(s_{2}-1\right)-1}}{\Gamma\left(-\left(s_{2}-1\right)\right)}-e_{2}\right]+\left(D_{t}^{s_{2}} e_{2}(t)\right) \frac{(t)^{-\left(s_{2}-1\right)-1}}{\Gamma\left(-\left(s_{2}-1\right)\right)}\right] \\
& +e_{3}\left[D _ { t } ^ { 1 - s _ { 3 } } \left(D_{t}^{s_{3}-1}\left[\tilde{b}_{3} z_{3}-\tilde{c}_{2} z_{2}+\tilde{d}_{2} x_{2}^{2}-b_{1} z_{1}-\left(D_{t}^{s_{3}-1} e_{3}(t)\right) \frac{(t)^{-\left(s_{3}-1\right)-1}}{\Gamma\left(-\left(s_{3}-1\right)\right)}-e_{3}\right]+\left(D_{t}^{s_{3}} e_{3}(t)\right)\right.\right. \\
& \left.\left.\times \frac{(\mathrm{t})^{-\left(s_{3}-1\right)-1}}{\Gamma\left(-\left(s_{3}-1\right)\right)}\right]+\tilde{\mathrm{a}}_{1} \dot{\tilde{\mathrm{a}}}_{1}+\tilde{\mathrm{b}}_{1} \dot{\tilde{\mathrm{b}}}_{1}+\tilde{\mathrm{c}}_{1} \dot{\tilde{\mathrm{c}}}_{1}+\tilde{\mathrm{a}}_{2} \dot{\tilde{\mathrm{a}}}_{2}+\tilde{\mathrm{b}}_{2} \dot{\tilde{\mathrm{b}}}_{2}+\tilde{\mathrm{c}}_{2} \dot{\tilde{\mathrm{c}}}_{2}+\tilde{\mathrm{d}}_{2} \dot{\tilde{\mathrm{d}}}_{2}+\tilde{\mathrm{a}}_{3} \dot{\tilde{\mathrm{a}}}_{3}+\tilde{\mathrm{b}}_{3} \dot{\tilde{\mathrm{b}}}_{3}+\tilde{\mathrm{c}}_{3} \dot{\tilde{\mathrm{c}}}_{3}\right),
\end{aligned}
$$

where $s \in[0,1],(1-s)>0$ and $(s-1)<0$. Now using (2.2) then (4.8) becomes

$$
\begin{aligned}
\dot{V}= & e_{1}\left[-\tilde{a}_{3}\left(y_{3}-x_{3}\right)+\tilde{a}_{2}\left(y_{2}-x_{2}\right)+\tilde{a}_{1}\left(y_{1}-x_{1}\right)-e_{1}\right]+e_{2}\left[-\left(\tilde{c}_{3}-\tilde{a}_{3}\right) x_{3}-\tilde{c}_{3} y_{3}\right. \\
& \left.+\tilde{b}_{2} x_{2}+\tilde{c}_{1} y_{1}-e_{2}\right]+e_{3}\left[\tilde{b}_{3} z_{3}-\tilde{c}_{2} z_{2}+\tilde{d}_{2} x_{2}^{2}-b_{1} z_{1}-e_{3}\right]+\tilde{a}_{1}\left(-\left(y_{1}-x_{1}\right) e_{1}\right) \\
& +\tilde{b}_{1}\left(z_{1} e_{3}\right)+\tilde{c}_{1}\left(-y_{1} e_{2}\right)+\tilde{a}_{2}\left(-\left(y_{1}-x_{1}\right) e_{1}\right)+\tilde{b}_{2}\left(-x_{1} e_{2}\right)+\tilde{c}_{2}\left(z_{2} e_{3}\right)+\tilde{d}_{2}\left(-x_{2}^{2} e_{3}\right) \\
& +\tilde{a}_{3}\left(\left(y_{3}-x_{3}\right) e_{1}-x_{3} e_{2}\right)+\tilde{b}_{3}\left(-z_{3} e_{3}\right)+\tilde{c}_{3}\left(\left(x_{3}+y_{3}\right) e_{2}\right)=-e^{\top} e \leqslant 0 .
\end{aligned}
$$

In light of the Lyapunov stability theory [20], the error dynamical system can converge to the origin asymptotically, which implies that the adaptive combined synchronization of fractional order chaotic systems with fully unknown parameters is achieved.

\subsection{Numerical simulations}

In the numerical results of the proposed modify adaptive combined synchronization, we use AdamsBashforth-Moulton method to solve the systems for the fractional order $s_{i}=0.95, i=1,2,3$, and the unknown parameters are chosen as $a_{1}=35, b_{1}=3, c_{1}=28$ and $a_{2}=10_{2}, b_{2}=40, c_{2}=2.5, d_{2}=4$ and $a_{3}=35, b_{3}=3, c_{3}=27$. The initial values of the fractional-order drive systems (4.1)-(4.2), the fractional-order response system (4.3) and the estimated parameters are arbitrarily chosen in simulations as $\left(x_{1}(0)=0.2, y_{1}(0)=0.6, z_{1}(0)=1\right),\left(x_{2}(0)=7, y_{2}(0)=11, z_{2}(0)=15\right),\left(x_{3}(0)=0.2, y_{3}(0)=0, z_{3}(0)=\right.$ $0.5)$, and $\hat{a}_{1}(0)=2.0, \hat{b}_{1}(0)=2.0, \hat{c}_{1}(0)=2.0, \hat{a}_{2}(0)=2.0, \hat{b}_{2}(0)=2.0, \hat{c}_{2}(0)=2.0, \hat{\mathrm{d}}_{2}(0)=2.0$ and $\hat{\mathrm{a}}_{3}(0)=2.0, \hat{\mathrm{b}}_{3}(0)=2.0, \hat{\mathrm{c}}_{3}(0)=2.0$, respectively. Combined synchronization of the systems (4.1)-(4.2) and (4.3) via adaptive control law (4.5) and (4.6) are shown in Figs. 1-2. Fig. 1 (a)-(c) displays the combined synchronization among three fractional order chaotic (4.1)-(4.2) and (4.3). Fig. 2 (a) display the combined synchronization errors, $e_{1}, e_{2}, e_{3}$ with time t. Fig. 2 (b)-(d) displays the time response of estimated values of parameters $\hat{a}_{1}, \hat{b}_{1}, \hat{c}_{1}, \hat{a}_{2}, \hat{b}_{2}, \hat{c}_{2}, \hat{d}_{2}$ of drive systems (4.1) and (4.2) and $\hat{a}_{3}, \hat{b}_{3}, \hat{c}_{3}$ of response system (4.3). 
(a)

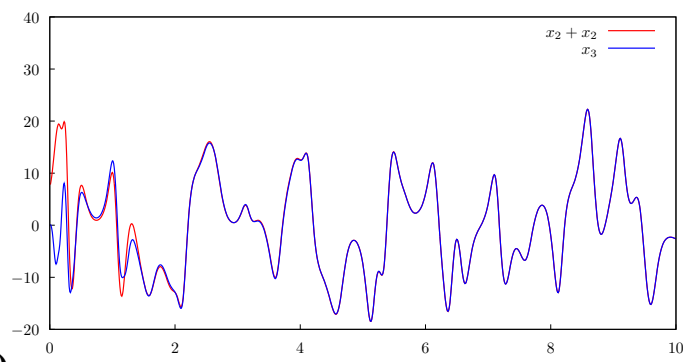

(b)
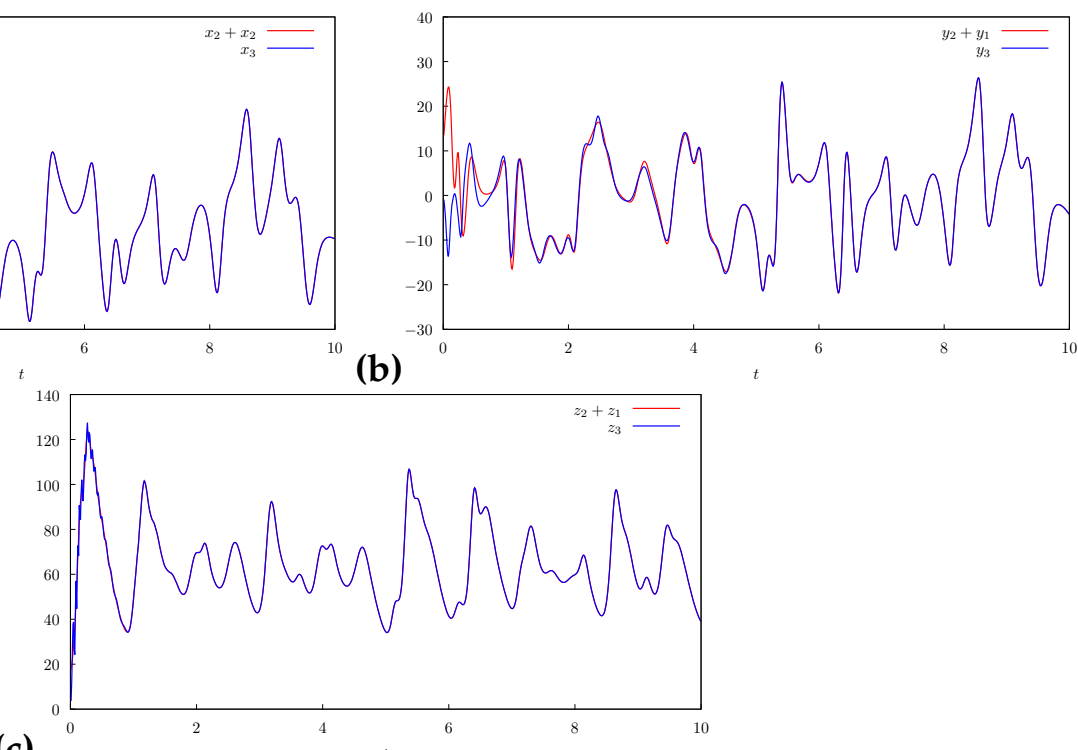

Figure 1: Combined synchronization among three fractional order chaotic (4.1)-(4.2) and (4.3): (a): Signals $x_{2}+x_{1}$ and $x_{3} ;(\mathbf{b})$ : signals $y_{2}+y_{1}$ and $y_{3} ;(\mathbf{c})$ signals $z_{2}+z_{1}$ and $z_{3}$.

(a)
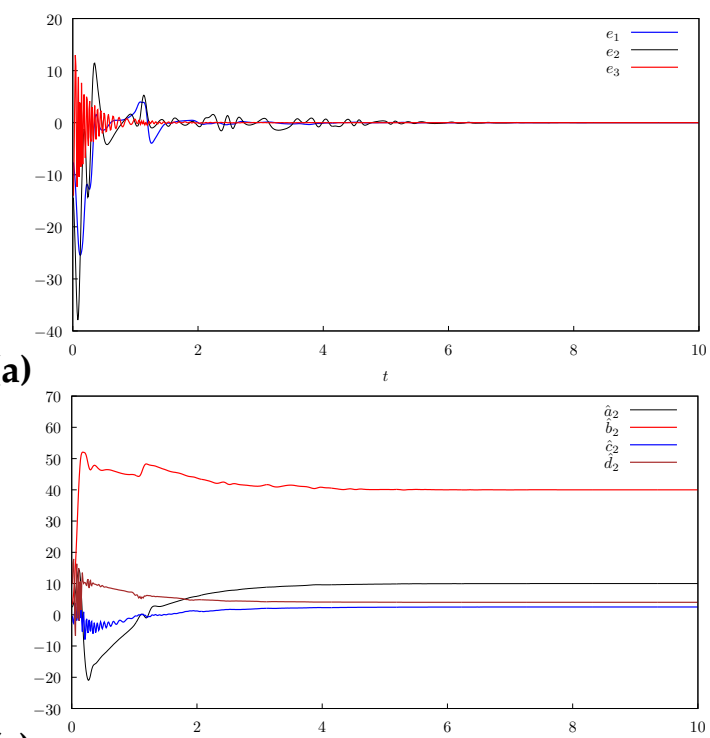

(c)

(d)

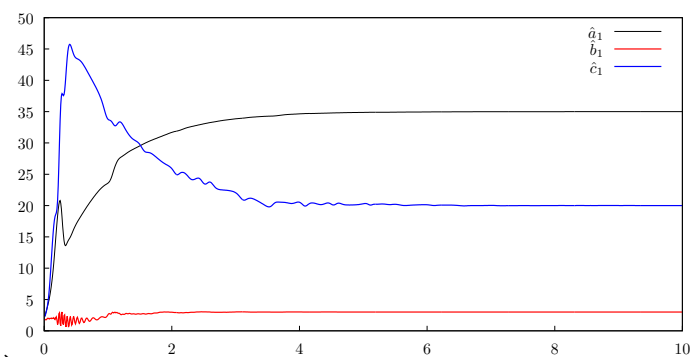

(b)

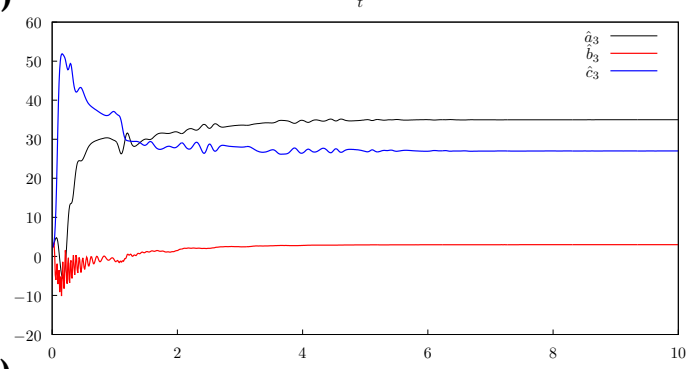

Figure 2: (a): Combined synchronization errors, $e_{1}, e_{2}, e_{3}$ with time $t$; (b)-(d): Time response of estimated values of parameters $\hat{\mathrm{a}}_{1}, \hat{\mathrm{b}}_{1}, \hat{\mathrm{c}}_{1}, \hat{\mathrm{a}}_{2}, \hat{\mathrm{b}}_{2}, \hat{\mathrm{c}}_{2}, \hat{\mathrm{d}}_{2}$ of drive systems (4.1) and (4.2) and $\hat{\mathrm{a}}_{3}, \hat{\mathrm{b}}_{3}, \hat{\mathrm{c}}_{3}$ of response system (4.3).

\section{Adaptive combined synchronization of Lü, Liu, Genesio-Tesi and Chen systems}

This section presents the the combined synchronization of Lü, Liu, Genesio-Tesi and Chen systems with unknown parameters, the Lü, Liu system, Genesio-Tesi $[13,36]$ are considers as the drive systems I, II,III respectively.

$$
\begin{array}{lll}
D_{t}^{s_{1}} x_{1}=a_{1}\left(y_{1}-x_{1}\right), & D_{t}^{s_{2}} y_{1}=-x_{1} z_{1}+c_{1} y_{1}, & D_{t}^{s_{3}} z_{1}=x_{1} y_{1}-b_{1} z_{1}, \\
D_{t}^{s_{1}} x_{2}=a_{2}\left(y_{2}-x_{2}\right), & D_{t}^{s_{2}} y_{2}=b_{2} x_{2}-x_{2} z_{2}, & D_{t}^{s_{3}} z_{2}=-c_{2} z_{2}+d_{2} x_{2}^{2}, \\
D_{t}^{s_{1}} x_{3}=y_{3}, & D_{t}^{s_{2}} y_{3}=z_{3}, & D_{t}^{s_{3}} z_{3}=-a_{3} x_{3}-b_{3} y_{3}-c_{3} z_{3}+m_{3} x_{3}^{2} .
\end{array}
$$


The Chen system (4.3) is taken as response system with control inputs $\mathfrak{u}_{1}, \mathfrak{u}_{2}, u_{3}$ as

$$
D_{t}^{s_{1}} x_{4}=a_{4}\left(y_{4}-x_{4}\right)+u_{1}, \quad D_{t}^{s_{1}} y_{4}=\left(c_{4}-a_{4}\right) x_{4}-x_{4} z+c_{4} y_{4}+u_{2}, \quad D_{t}^{s_{1}} z_{4}=x_{4} y_{4}-b_{4} z_{4}+u_{3} .
$$

where $\mathrm{U}=\left(\mathfrak{u}_{1}, \mathfrak{u}_{2}, \mathfrak{u}_{3}\right)^{\top}$ denote to the controller function. In the following, an effective adaptive controller function is constructed to achieve the combined synchronization between Lü, Liu, Gensio systems and Chen system with fully unknown parameters. By subtracting (5.1)-(5.3) from (5.4) the following error system is obtained:

$$
\begin{aligned}
& D_{t}^{s_{1}} e_{1}(t)=a_{4}\left(y_{4}-x_{4}\right)-y_{3}-a_{2}\left(y_{2}-x_{2}\right)-a_{1}\left(y_{1}-x_{1}\right)+u_{1}, \\
& D_{t}^{s_{2}} e_{2}(t)=\left(c_{4}-a_{4}\right) x_{4}-x_{4} z+c_{4} y_{4}-z_{3}-b_{2} x_{2}+x_{2} z_{2}+x_{1} z_{1}-c_{1} y_{1}+u_{2}, \\
& D_{t}^{s_{3}} e_{3}(t)=x_{4} y_{4}-b_{4} z_{4}+a_{3} x_{3}+b_{3} y_{3}+c_{3} z_{3}-m_{3} x_{3}^{2}+c_{2} z_{2}-d_{2} x_{2}^{2}-x_{1} y_{1}+b_{1} z_{1}+u_{3},
\end{aligned}
$$

where $e_{1}=x_{3}-x_{2}-x_{1}, e_{2}=y_{3}-y_{2}-y_{1}$ and $e_{3}=z_{3}-z_{2}-z_{1}$. Our objective is to design the controller $\mathrm{U}$ with a parameter update law in order to achieve the globally and asymptotically combined synchronization between (5.1)-(5.3) and (5.4). In order to show that systems (5.1)-(5.3) and (5.4) are combined synchronized, the following Theorem is construct.

Theorem 5.1. The asymptotically combined synchronization of the systems (5.1)-(5.3) and the system (5.4) will achieve for any distinct initial condition with following modify adaptive controller

$$
\begin{aligned}
u_{1}= & -a_{4}\left(y_{4}-x_{4}\right)+a_{2}\left(y_{2}-x_{2}\right)+a_{1}\left(y_{1}-x_{1}\right)+y_{3}+D_{t}^{s_{1}-1}\left[-\hat{a}_{4}\left(y_{4}-x_{4}\right)\right. \\
& \left.+\hat{a}_{2}\left(y_{2}-x_{2}\right)+\hat{a}_{1}\left(y_{1}-x_{1}\right)-\left(D_{t}^{s_{1}-1} e_{1}(t)\right) \frac{(t)^{-\left(s_{1}-1\right)-1}}{\Gamma\left(-\left(s_{1}-1\right)\right)}-e_{1}\right] \\
u_{2}= & -\left(c_{4}-a_{4}\right) x_{4}+x_{4} z_{4}-c_{4} y_{4}+z_{3}-x_{2} z_{2}+b_{2} x_{2}+c_{1} y_{1}-x_{1} z_{1} \\
& +D_{t}^{s_{2}-1}\left[-\left(\hat{c}_{4}-\hat{a}_{4}\right) x_{4}-\hat{c}_{4} y_{4}+\hat{b}_{2} x_{2}+\hat{c}_{1} y_{1}-\left(D_{t}^{s_{2}-1} e_{2}(t)\right) \frac{(t)^{-\left(s_{2}-1\right)-1}}{\Gamma\left(-\left(s_{2}-1\right)\right)}-e_{2}\right], \\
u_{3}= & -x_{4} y_{4}+b_{4} z_{4}-a_{3} x_{3}-b_{3} y_{3}-c_{3} z_{3}+m_{3} x_{3}^{2}-c_{2} z_{2}+d_{2} x_{2}^{2}-b_{1} z_{1}+x_{1} y_{1} \\
& +D_{t}^{s_{3}-1}\left[\hat{b}_{4} z_{4}-\hat{a}_{3} x_{3}-\hat{b}_{3} y_{3}-\hat{c}_{3} z_{3}+\hat{m}_{3} x_{3}^{2}-\hat{c}_{2} z_{2}+\hat{d}_{2} x_{2}^{2}-\hat{b}_{1} z_{1}\right. \\
& \left.-\left(D_{t}^{s_{3}-1} e_{3}(t)\right) \frac{(t)^{-\left(s_{3}-1\right)-1}}{\Gamma\left(-\left(s_{3}-1\right)\right)}-e_{3}\right]
\end{aligned}
$$

and the update rule of the unknown parameters are taken as

$$
\begin{aligned}
& \dot{\tilde{\mathrm{a}}}_{1}=-\left(\mathrm{y}_{1}-\mathrm{x}_{1}\right) \mathrm{e}_{1}, \quad \dot{\tilde{\mathrm{b}}}_{1}=z_{1} e_{3}, \quad \dot{\tilde{\mathrm{c}}}_{1}=-\mathrm{y}_{1} \mathrm{e}_{2}, \quad \dot{\tilde{\mathrm{a}}}_{2}=-\left(\mathrm{y}_{2}-\mathrm{x}_{2}\right) \mathrm{e}_{1}, \quad \dot{\tilde{\mathrm{b}}}_{2}=-\mathrm{x}_{2} \mathrm{e}_{2}, \\
& \dot{\tilde{c}}_{2}=z_{2} e_{3}, \quad \quad \dot{\tilde{d}}_{2}=-x_{2}^{2} e_{3}, \quad \dot{\tilde{a}}_{3}=x_{3} e_{3}, \quad \dot{\tilde{\mathrm{b}}}_{3}=y_{3} e_{3}, \quad \dot{\tilde{c}}_{3}=z_{3} e_{3}, \\
& \dot{\tilde{m}}_{3}=-x_{2}^{2} e_{3}, \quad \dot{\tilde{a}}_{4}=\left(y_{4}-x_{4}\right) e_{1}-x_{4} e_{2}, \quad \dot{\tilde{b}}_{4}=-z_{4} e_{3}, \quad \dot{\tilde{c}}_{4}=\left(x_{4}+y_{4}\right) e_{2},
\end{aligned}
$$

where $\hat{a}_{1}, \hat{b}_{1}, \hat{c}_{1}, \hat{a}_{2}, \hat{b}_{2}, \hat{c}_{2}, \hat{d}_{2}, \hat{a}_{3}, \hat{b}_{3}, \hat{c}_{3}, \hat{m}_{3}, \hat{a}_{4}, \hat{b}_{4}, \hat{c}_{4}$ are estimates of $a_{1}, b_{1}, c_{1}, a_{2}, b_{2}, c_{2}, d_{2}, a_{3}, b_{3}, c_{3}, m_{3}, a_{4}, b_{4}$, $\mathrm{c}_{4}$, respectively.

Proof. By applying the modify adaptive controller (5.6) to (5.5), the following new error systems is obtained:

$$
\begin{aligned}
& D_{t}^{s_{1}} e_{1}(t)=D_{t}^{s_{1}-1}\left[-\tilde{a}_{4}\left(y_{4}-x_{4}\right)+\tilde{a}_{2}\left(y_{2}-x_{2}\right)+\tilde{a}_{1}\left(y_{1}-x_{1}\right)-\left(D_{t}^{s_{1}-1} e_{1}(t)\right) \frac{(t)^{-\left(s_{1}-1\right)-1}}{\Gamma\left(-\left(s_{1}-1\right)\right)}-e_{1}\right], \\
& D_{t}^{s_{2}} e_{2}(t)=D_{t}^{s_{2}-1}\left[-\left(\tilde{c}_{4}-\tilde{a}_{4}\right) x_{4}-\tilde{c}_{4} y_{4}+\tilde{b}_{2} x_{2}+\tilde{c}_{1} y_{1}-\left(D_{t}^{s_{2}-1} e_{2}(t)\right) \frac{(t)^{-\left(s_{2}-1\right)-1}}{\Gamma\left(-\left(s_{2}-1\right)\right)}-e_{2}\right],
\end{aligned}
$$




$$
\begin{aligned}
\mathrm{D}_{\mathrm{t}}^{s_{3}} e_{3}(\mathrm{t})= & \mathrm{D}_{\mathrm{t}}^{\mathrm{s}_{3}-1}\left[\tilde{\mathrm{b}}_{4} z_{4}-\tilde{\mathrm{a}}_{3} x_{3}-\tilde{\mathrm{b}}_{3} \mathrm{y}_{3}-\tilde{\mathrm{c}}_{3} z_{3}+\tilde{\mathrm{m}}_{3} x_{3}^{2}-\tilde{\mathrm{c}}_{2} z_{2}+\tilde{\mathrm{d}}_{2} x_{2}^{2}-\tilde{\mathrm{b}}_{1} z_{1}\right. \\
& \left.-\left(\mathrm{D}_{\mathrm{t}}^{\mathrm{s}_{3}-1} e_{3}(\mathrm{t})\right) \frac{(\mathrm{t})^{-\left(s_{3}-1\right)-1}}{\Gamma\left(-\left(\mathrm{s}_{3}-1\right)\right)}-e_{3}\right]
\end{aligned}
$$

where $\tilde{a}_{1}=\hat{a}_{1}-a_{1}, \tilde{b}_{1}=\hat{b}_{1}-b_{1}, \tilde{c}_{1}=\hat{c}_{1}-c_{1}, \tilde{a}_{2}=\hat{a}_{2}-a_{2}, \tilde{b}_{2}=\hat{b}_{2}-b_{2}, \tilde{c}_{2}=\hat{c}_{2}-c_{2}, \tilde{d}_{2}=\hat{d}_{2}-d_{2}, \tilde{a}_{3}=$ $\hat{a}_{3}-a_{3}, \tilde{b}_{3}=\hat{b}_{3}-b_{3}, \tilde{c}_{3}=\hat{c}_{3}-c_{3}, \tilde{m}_{3}=\hat{m}_{3}-m_{3}, \tilde{a}_{4}=\hat{a}_{4}-a_{4}, \tilde{b}_{4}=\hat{b}_{4}-b_{4}, \tilde{c}_{4}=\hat{c}_{4}-c_{4}$. Construct a Lyapunov function candidate as

$$
\mathrm{V}=\frac{1}{2}\left(e^{\top} e+\tilde{\mathrm{a}}_{1}^{2}+\tilde{\mathrm{b}}_{1}^{2}+\tilde{\mathrm{c}}_{1}^{2}+\tilde{\mathrm{a}}_{2}^{2}+\tilde{\mathrm{b}}_{2}^{2}+\tilde{\mathrm{c}}_{2}^{2}+\tilde{\mathrm{d}}_{2}^{2}+\tilde{\mathrm{a}}_{3}^{2}+\tilde{\mathrm{b}}_{3}^{2}+\tilde{\mathrm{c}}_{3}^{2}+\tilde{\mathrm{m}}_{3}^{2}+\tilde{\mathrm{a}}_{4}^{2}+\tilde{\mathrm{b}}_{4}^{2}+\tilde{\mathrm{c}}_{4}^{2}\right) .
$$

Differentiate $V$ with respect to time the following is obtained:

$$
\begin{aligned}
& \dot{V}=\left(e^{\top} \dot{\mathrm{e}}+\tilde{\mathrm{a}}_{1} \dot{\tilde{\mathrm{a}}}_{1}+\tilde{\mathrm{b}}_{1} \dot{\tilde{\mathrm{b}}}_{1}+\tilde{\mathrm{c}}_{1} \dot{\tilde{\mathrm{c}}}_{1}+\tilde{\mathrm{a}}_{2} \dot{\tilde{\mathrm{a}}}_{2}+\tilde{\mathrm{b}}_{2} \dot{\tilde{\mathrm{b}}}_{2}+\tilde{\mathrm{c}}_{2} \dot{\tilde{\mathrm{c}}}_{2}+\tilde{\mathrm{d}}_{2} \dot{\tilde{\mathrm{d}}}_{2}+\tilde{\mathrm{a}}_{3} \dot{\tilde{\mathrm{a}}}_{3}\right. \\
& \left.+\tilde{\mathrm{b}}_{3} \dot{\tilde{\mathrm{b}}}_{3}+\tilde{\mathrm{c}}_{3} \dot{\tilde{\mathrm{c}}}_{3}+\tilde{\mathrm{m}}_{3} \dot{\tilde{\mathrm{m}}}_{3}+\tilde{\mathrm{a}}_{4} \dot{\tilde{\mathrm{a}}}_{4}+\tilde{\mathrm{b}}_{4} \dot{\tilde{\mathrm{b}}}_{4}+\tilde{\mathrm{c}}_{4} \dot{\tilde{\mathrm{c}}}_{4}\right) .
\end{aligned}
$$

Using (2.1) in (5.8) we get

$$
\begin{aligned}
& \dot{V}=\left(e_{1}\left[D_{t}^{1-s_{1}}\left(D_{t}^{s_{1}} e_{1}(t)\right)+\left(D_{t}^{s_{1}} e_{1}(t)\right) \frac{(t)^{-\left(s_{1}-1\right)-1}}{\Gamma\left(-\left(s_{1}-1\right)\right)}\right]+e_{2}\left[D_{t}^{1-s_{2}}\left(D_{t}^{s_{2}} e_{2}(t)\right)\right.\right. \\
& \left.+\left(D_{t}^{s_{2}} e_{2}(t)\right) \frac{(t)^{-\left(s_{2}-1\right)-1}}{\Gamma\left(-\left(s_{2}-1\right)\right)}\right]+e_{3}\left[D_{t}^{1-s_{3}}\left(D_{t}^{s_{3}} e_{3}(t)\right)+\left(D_{t}^{s_{3}} e_{3}(t)\right) \frac{(t)^{-\left(s_{3}-1\right)-1}}{\Gamma\left(-\left(s_{3}-1\right)\right)}\right] \\
& +\tilde{\mathrm{a}}_{1} \dot{\tilde{\mathrm{a}}}_{1}+\tilde{\mathrm{b}}_{1} \dot{\tilde{\mathrm{b}}}_{1}+\tilde{\mathrm{c}}_{1} \dot{\tilde{\mathrm{c}}}_{1}+\tilde{\mathrm{a}}_{2} \dot{\tilde{\mathrm{a}}}_{2}+\tilde{\mathrm{b}}_{2} \dot{\tilde{\mathrm{b}}}_{2}+\tilde{\mathrm{c}}_{2} \dot{\tilde{\mathrm{c}}}_{2}+\tilde{\mathrm{d}}_{2} \dot{\tilde{\mathrm{d}}}_{2}+\tilde{\mathrm{a}}_{3} \dot{\tilde{\mathrm{a}}}_{3}+\tilde{\mathrm{b}}_{3} \dot{\tilde{\mathrm{b}}}_{3}+\tilde{\mathrm{c}}_{3} \dot{\tilde{\mathrm{c}}}_{3}+\tilde{\mathrm{m}}_{3} \dot{\tilde{\mathrm{m}}}_{3} \\
& \left.+\tilde{\mathrm{a}}_{4} \dot{\tilde{\mathrm{a}}}_{4}+\tilde{\mathrm{b}}_{4} \dot{\tilde{\mathrm{b}}}_{4}+\tilde{\mathrm{c}}_{4} \dot{\tilde{\mathrm{c}}}_{4}\right) \\
& =\left(e _ { 1 } \left[D _ { t } ^ { 1 - q _ { 1 } } \left(D _ { t } ^ { q _ { 1 } - 1 } \left[-\tilde{a}_{4}\left(y_{4}-x_{4}\right)+\tilde{a}_{2}\left(y_{2}-x_{2}\right)+\tilde{a}_{1}\left(y_{1}-x_{1}\right)\right.\right.\right.\right. \\
& \left.\left.-\left(D_{t}^{q_{1}-1} e_{1}(t)\right) \frac{(t)^{-\left(s_{1}-1\right)-1}}{\Gamma\left(-\left(s_{1}-1\right)\right)}-e_{1}\right]+\left(D_{t}^{s_{1}} e_{1}(t)\right) \frac{(t)^{-\left(s_{1}-1\right)-1}}{\Gamma\left(-\left(s_{1}-1\right)\right)}\right] \\
& +e_{2}\left[D _ { t } ^ { 1 - s _ { 2 } } \left(D _ { t } ^ { s _ { 2 } - 1 } \left[-\left(\tilde{c}_{4}-\tilde{a}_{4}\right) x_{4}-\tilde{c}_{4} y_{4}+\tilde{b}_{2} x_{2}+\tilde{c}_{1} y_{1}\right.\right.\right. \\
& \left.\left.-\left(D_{t}^{s_{2}-1} e_{2}(t)\right) \frac{(t)^{-\left(s_{2}-1\right)-1}}{\Gamma\left(-\left(s_{2}-1\right)\right)}-e_{2}\right]+\left(D_{t}^{s_{2}} e_{2}(t)\right) \frac{(t)^{-\left(s_{2}-1\right)-1}}{\Gamma\left(-\left(s_{2}-1\right)\right)}\right] \\
& +e_{3}\left[D _ { t } ^ { 1 - s _ { 3 } } \left(D _ { t } ^ { s _ { 3 } - 1 } \left[\tilde{b}_{4} z_{4}-\tilde{a}_{3} x_{3}-\tilde{b}_{3} y_{3}-\tilde{c}_{3} z_{3}+\tilde{m}_{3} x_{3}^{2}-\tilde{c}_{2} z_{2}+\tilde{d}_{2} x_{2}^{2}-\tilde{b}_{1} z_{1}\right.\right.\right. \\
& \left.\left.-\left(D_{t}^{s_{3}-1} e_{3}(t)\right) \frac{(t)^{-\left(s_{3}-1\right)-1}}{\Gamma\left(-\left(s_{3}-1\right)\right)}-e_{3}\right]+\left(D_{t}^{s_{3}} e_{3}(t)\right) \frac{(t)^{-\left(s_{3}-1\right)-1}}{\Gamma\left(-\left(s_{3}-1\right)\right)}\right]+\tilde{a}_{1} \dot{\tilde{a}}_{1}+\tilde{\mathrm{b}}_{1} \dot{\tilde{b}}_{1}
\end{aligned}
$$

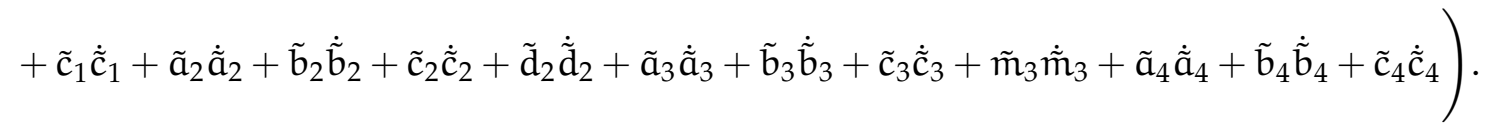

Now using (2.2), (5.9) reduces to

$$
\dot{V}=e_{1}\left[-\tilde{a}_{4}\left(y_{4}-x_{4}\right)+\tilde{a}_{2}\left(y_{2}-x_{2}\right)+\tilde{a}_{1}\left(y_{1}-x_{1}\right)-e_{1}\right]+e_{2}\left[-\left(\tilde{c}_{4}-\tilde{a}_{4}\right) x_{4}\right.
$$




$$
\begin{aligned}
& \left.-\tilde{c}_{4} y_{4}+\tilde{b}_{2} x_{2}+\tilde{c}_{1} y_{1}-e_{2}\right]+e_{3}\left[\tilde{b}_{4} z_{4}-\tilde{a}_{3} x_{3}-\tilde{b}_{3} y_{3}-\tilde{c}_{3} z_{3}+\tilde{m}_{3} x_{3}^{2}-\tilde{c}_{2} z_{2}\right. \\
& \left.+\tilde{d}_{2} x_{2}^{2}-\tilde{b}_{1} z_{1}-e_{3}\right]+\tilde{a}_{1}\left(-\left(y_{1}-x_{1}\right) e_{1}\right)+\tilde{b}_{1}\left(z_{1} e_{3}\right)+\tilde{c}_{1}\left(-y_{1} e_{2}\right)+\tilde{a}_{2}\left(-\left(y_{2}-x_{2}\right) e_{1}\right) \\
& +\tilde{b}_{2}\left(-x_{2} e_{2}\right)+\tilde{c}_{2}\left(z_{2} e_{3}\right)+\tilde{d}_{2}\left(-x_{2}^{2} e_{3}\right)+\tilde{a}_{3}\left(x_{3} e_{3}\right)+\tilde{b}_{3}\left(y_{3} e_{3}\right)+\tilde{c}_{3}\left(z_{3} e_{3}\right) \\
& +\tilde{m}_{3}\left(-x_{2}^{2} e_{3}\right)+\tilde{a}_{4}\left(\left(y_{4}-x_{4}\right) e_{1}-x_{4} e_{2}\right)+\tilde{b}_{4}\left(-z_{4} e_{3}\right)+\tilde{c}_{4}\left(\left(x_{4}+y_{4}\right) e_{2}\right) \\
& =-e^{\top} e \leqslant 0 .
\end{aligned}
$$

In light of the Lyapunov stability theory [20], the error dynamical system can converge to the origin asymptotically, which implies that the adaptive combined synchronization of fractional order chaotic systems with fully unknown parameters is achieved.

\subsection{Numerical simulations}

In the numerical results of the proposed modify adaptive combined synchronization, we use AdamsBashforth-Moulton method to solve the systems for the fractional order $s_{i}=0.95, i=1,2,3$, and the unknown parameters are chosen as $a_{1}=35, b_{1}=3, c_{1}=28$ and $a_{2}=35, b_{2}=3, c_{2}=28, a_{2}=$ $10_{2}, \mathrm{~b}_{2}=40, \mathrm{c}_{2}=2.5, \mathrm{~d}_{2}=4, \mathrm{a}_{3}=6, \mathrm{~b}_{3}=2.92, \mathrm{c}_{3}=1.2, \mathrm{~m}_{3}=1$ and $\mathrm{a}_{4}=35, \mathrm{~b}_{4}=3, \mathrm{c}_{4}=27$. The initial values of the fractional-order drive systems (5.1)-(5.3), the fractional-order response system (4.3) and the estimated parameters are arbitrarily chosen in simulations as $\left(x_{1}(0)=0.2, y_{1}(0)=0.6, z_{1}(0)=\right.$ $1),\left(x_{2}(0)=7, y_{2}(0)=11, z_{2}(0)=15\right),\left(x_{3}(0)=-3, y_{3}(0)=3, z_{3}(0)=5\right),\left(x_{4}(0)=0.2, y_{4}(0)=0, z_{4}(0)=0.5\right)$, and $\hat{a}_{1}(0)=2.0, \hat{b}_{1}(0)=2.0, \hat{c}_{1}(0)=2.0, \hat{a}_{2}(0)=2.0, \hat{b}_{2}(0)=2.0, \hat{c}_{2}(0)=2.0, \hat{\mathrm{d}}_{2}(0)=2.0, \hat{\mathrm{a}}_{3}(0)=$ $2.0, \hat{b}_{3}(0)=2.0, \hat{c}_{3}(0)=2.0, \hat{m}_{3}(0)=2.0$ and $\hat{a}_{4}(0)=2.0, \hat{b}_{4}(0)=2.0, \hat{c}_{4}(0)=2.0$, respectively. Combined synchronization among four fractional order chaotic (5.1)-(5.3) and (5.4) via adaptive control law (5.6) and (5.7) are shown in Figs. 3-4. Fig. 3 (a)-(c) displays the combined synchronization among three fractional order chaotic (5.1)-(5.3) and (5.4). Fig. 3 (d) displays the combined synchronization errors, $e_{1}, e_{2}, e_{3}$ with time t. Fig. 4 (a)-(d) displays the time response of estimated values of parameters $\hat{a}_{1}, \hat{b}_{1}, \hat{c}_{1}, \hat{a}_{2}, \hat{b}_{2}, \hat{c}_{2}, \hat{d}_{2}, \hat{a}_{3}, \hat{b}_{3}, \hat{c}_{3}, \hat{m}_{3}$ of drive systems (5.1)-(5.3) and $\hat{a}_{4}, \hat{b}_{4}, \hat{c}_{4}$ of response system (5.4).

(a)
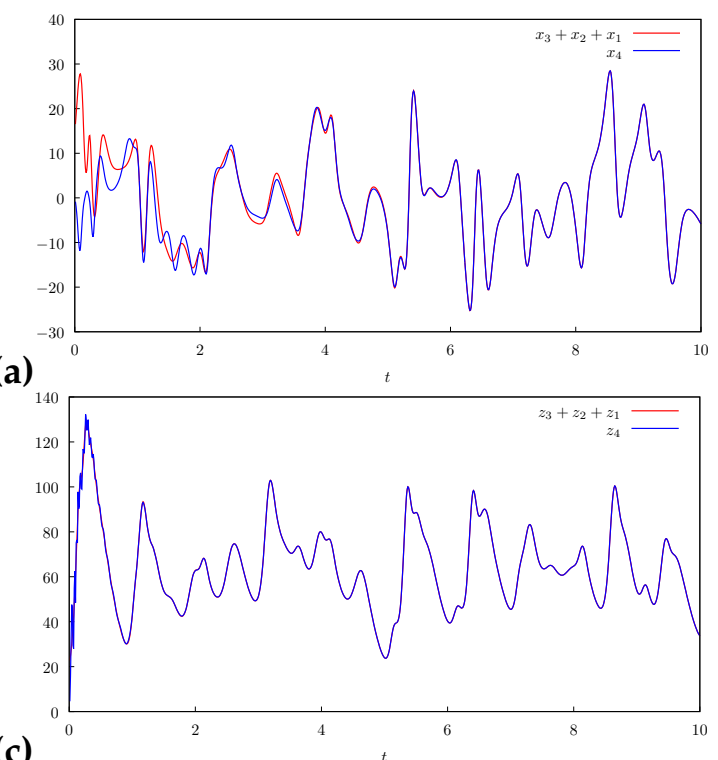

(b)
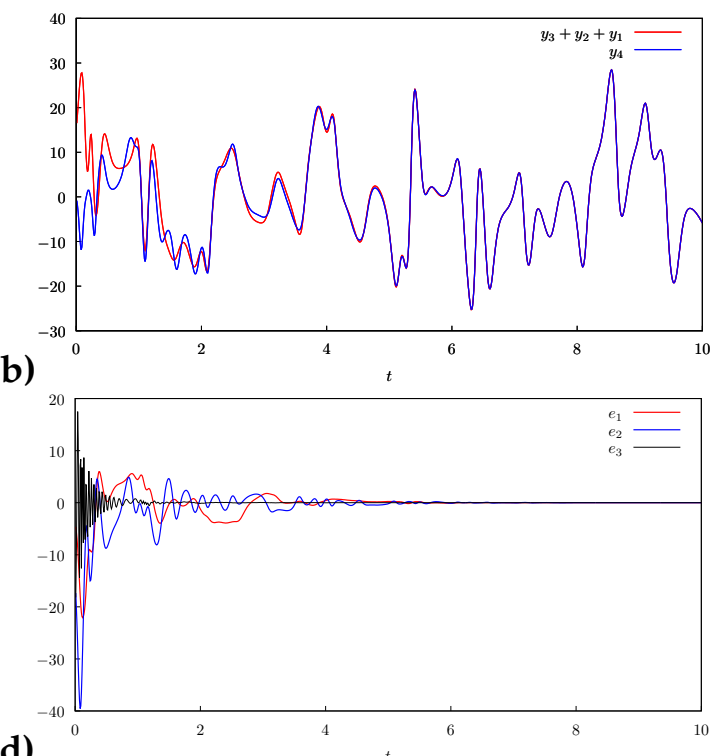

Figure 3: Combined synchronization among four fractional order chaotic (5.1)-(5.3) and (5.4): (a): signals $x_{3}+x_{2}+x_{1}$ and $x_{4}$; (b): signals $y_{3}+y_{2}+y_{1}$ and $y_{4} ;$ (c): signals $z_{3}+z_{2}+z_{1}$ and $z_{4} ;(d)$ : combined synchronization errors, $e_{1}, e_{2}, e_{3}$ with time $t$. 
(a)

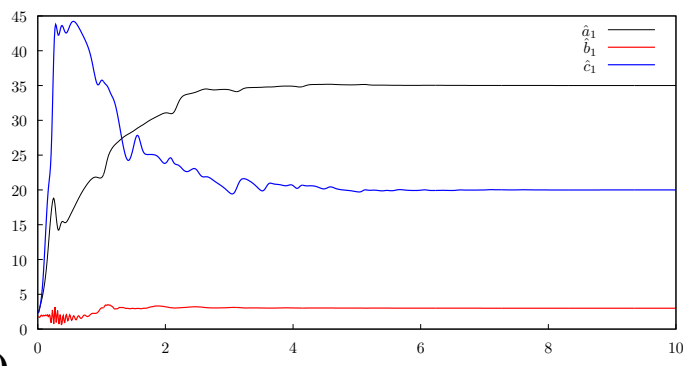

(c)

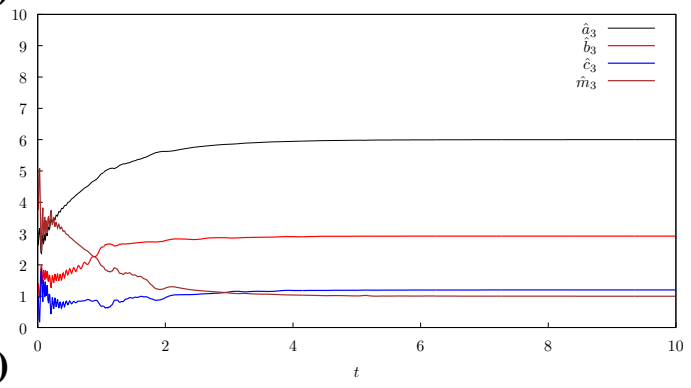

(b)

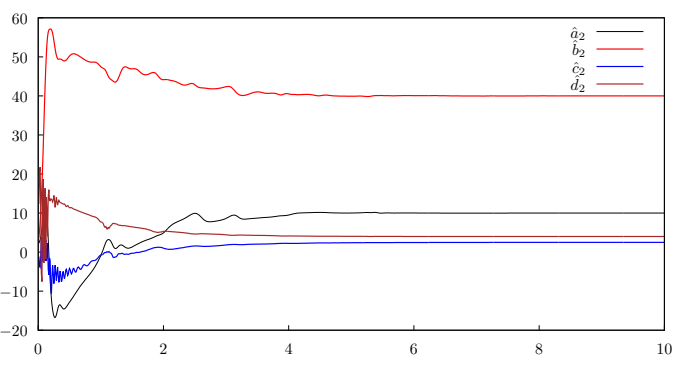

(d)

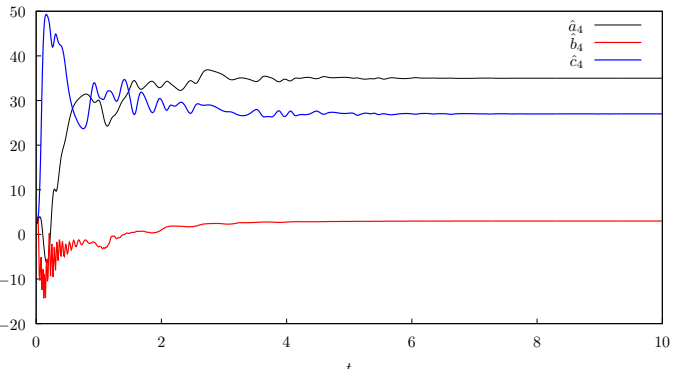

Figure 4: (a)-(d): Time response of estimated values of parameters $\hat{a}_{1}, \hat{b}_{1}, \hat{c}_{1}, \hat{a}_{2}, \hat{b}_{2}, \hat{c}_{2}, \hat{d}_{2}, \hat{a}_{3}, \hat{b}_{3}, \hat{c}_{3}, \hat{m}_{3}$ of drive systems (5.1), (5.2) and (5.3) $\hat{a}_{4}, \hat{b}_{4}, \hat{c}_{4}$ of response system (5.4).

\section{Conclusion}

This article presents a generalized synchronization scheme to study the adaptive combined synchronization of different fractional order chaotic systems with unknown parameters. The Lyapunov stability theory and the design of a nonlinear controller establish the asymptotic stability of the synchronization errors at the origin. Accordingly, suitable parameters updated laws estimate the true value of unknown parameters. Two numerical examples are illustrated and computer based simulation results are provided to verify the effectiveness of the proposed adaptive combined synchronization control approach. The proposed adaptive combination synchronization strategy is simple and generalized. The proposed adaptive combined synchronization strategy can be useful in secure communication systems.

\section{Acknowledgment}

We would like to extend our sincere thanks to deanship of academic research, vice presidency for graduate Studies and Scientific Research, University of Ha'il, Saudi Arabia, for funding this research. Grant number BA-1916.

\section{References}

[1] S. K. Agrawal, S. Das, A modified adaptive control method for synchronization of some fractional chaotic systems with unknown parameters, Nonlinear Dynam., 73 (2013), 907-919. 1, 2

[2] S. K. Agrawal, S. Das, Function projective synchronization between four dimensional chaotic systems with uncertain parameters using modified adaptive control method, J. Process Control, 24 (2014), 517-530.

[3] S. K. Agrawal, M. Srivastava, S. Das, Synchronization of fractional order chaotic systems using active control method, Chaos Solitons Fractals, 45 (2012), 737-752.

[4] I. Ahmad, A. B. Saaban, A. B. Ibrahim, M. Shahzad, Global chaos synchronization of new chaotic system using linear active control, Complexity, 21 (2015), 379-386.

[5] I. Ahmad, A. B. Saaban, A. B. Ibrahim, M. Shahzad, N. Naveed, The synchronization of chaotic systems with different dimensions by a robust generalized active control, Optik, 127 (2016), 4859-4871. 1

[6] I. Ahmad, M. Shafiq, M. M. Al-Sawalha, Globally exponential multi switching-combination synchronization control of chaotic systems for secure communications, Chinese J. Phys., 56 (2018), 974-987. 1

[7] I. Ahmad, M. Shafiq, M. Shahzad, Global Finite-Time Multi-Switching Synchronization of Externally Perturbed Chaotic Oscillators, Circuits Systems Signal Process., 37 (2018), 5253-5278. 1 
[8] M. M. Al-Sawalha, A. Al-Sawalha, Anti-synchronization of fractional order chaotic and hyperchaotic systems with fully unknown parameters using modified adaptive control, Open Phys., 14 (2016), 304-313. 4

[9] M. M. Al-Sawalha, M. Shoaib, Reduced-order synchronization of fractional order chaotic systems with fully unknown parameters using modified adaptive control, J. Nonlinear Sci. Appl., 9 (2016), 1815-1825. 1

[10] L. Y. T. Andrew, L. X. Feng, C. Y. Dong, Z. Hui, A novel adaptive-impulsive synchronization of fractional-order chaotic systems, Chinese Phys. B, 24 (2015), 7 pages.

[11] S. Bhalekar, V. D. Gejji, Synchronization of different fractional order chaotic systems using active control, Commun. Nonlinear. Sci. Numer. Simulat., 15 (2010), 3536-3546.

[12] D. Chen, R. F. Zhang, J. C. Sprott, H. T. Chen, X. Y. Ma, Synchronization between integer-order chaotic systems and a class of fractional-order chaotic systems via sliding mode control, Chaos, 22 (2012), 9 pages. 1

[13] M. R. Faieghi, H. Delavari, Chaos in fractional-order Genesio-Tesi system and its synchronization, Commun. Nonlinear Sci. Numer. Simul., 17 (2011), 7317-7341. 5

[14] Z. Gao, X. Z. Liao, Integral sliding mode control for fractional-order systems with mismatched uncertainties, Nonlinear Dynam., 72 (2013), 27-35. 1

[15] F. Gao, H. M. Srivastava, Y. N. Gao, X.-J. Yang, A coupling method involving the Sumudu transform and the variational iteration method for a class of local fractional diffusion equations, J. Nonlinear Sci. Appl., 9 (2016), 5830-5835. 1

[16] A. K. Golmankhaneh, R. Arefi, D. Baleanu, Synchronization in a nonidentical fractional order of a proposed modified system, J. Vib. Control, 21 (2015), 1154-1161. 1

[17] A. Hajipour, S. S. Aminabadi, Synchronization of chaotic Arneodo system of incommensurate fractional order with unknown parameters using adaptive method, Optik, 127 (2016), 7704-7709. 1

[18] A. S. Hegazi, E. Ahmed, A. E. Matouk, On chaos control and synchronization of the commensurate fractional order Liu system, Commun. Nonlinear. Sci. Numer. Simul., 18 (2013), 1193-1202. 4

[19] R. Hilfer, Application of fractional Calculus in Physics, World Scientific Publishing Co., River Edge, (2000). 1

[20] A. M. Liapunov, Stability of Motion, Academic Press, New York-London, (1966). 1, 3, 4, 5

[21] W. Y. Ma, C. P. Li, Y. J. Wu, Impulsive synchronization of fractional Takagi-Sugeno fuzzy complex networks, Chaos, 26 (2016), 8 pages. 1

[22] A. A. Othman, M. S. M. Noorani, M. M. Al-Sawalha, Adaptive dual anti-synchronization of chaotic systems with fully uncertain parameters, Optik, 127 (2016), 10478-10489. 1

[23] A. A. Othman, M. S. M. Noorani, M. M. Al-Sawalha, Adaptive dual synchronization of chaotic and hyperchaotic systems with fully uncertain parameters, Optik, 127 (2016), 7852-7864.

[24] A. A. Othman, M. S. M. Noorani, M. M. Al-Sawalha, Dual synchronization of chaotic and hyperchaotic systems, J. Nonlinear Sci. Appl., 9 (2016), 4666-4677.

[25] A. A. Othman, M. S. M. Noorani, M. M. Al-Sawalha, Function projective dual synchronization of chaotic systems with uncertain parameters, Nonlinear Dyn. Syst. Theory, 17 (2017), 193-204. 1

[26] L. M. Pecora, T. L. Carroll, Synchronization in chaotic systems, Phys. Rev. Lett., 64 (1990), 821-824. 1

[27] I. Podlubny, Fractional Differential Equations, Acedemic Press, San Diego, (1999). 1, 2

[28] M. Pourmahmood Aghababa, Control of non-linear non-integer-order systems using variable structure control theory, Trans. Inst. Measurement Control, 36 (2014), 425-432. 1

[29] M. Pourmahmood Aghababa, H. Feizi, Design of a sliding mode controller for synchronizing chaotic systems with parameter and model uncertainties and external disturbances, Trans. Inst. Measurement Control, 34 (2012), 990-997.

[30] A. G. Radwan, K. Moaddy, K. N. Salama, S. Momani, I. Hashim, Control and switching synchronization of fractional order chaotic systems using active control technique, J. Adv. Res., 5 (2014), 125-132. 1

[31] K. Sayevand, K. Pichaghchi, Analysis of nonlinear fractional KdV equation based on He's fractional derivative, Nonlinear Sci. Lett. A, 7 (2016), 77-85. 1

[32] M. Shafiq, I. Ahmad, Multi-Switching Combination Anti-synchronization of Unknown Hyperchaotic Systems, Arab. J. Sci. Eng., 44 (2019), 7335-7350. 1

[33] A. K. Singh, V. K. Yadav, S. Das, Dual combination synchronization of the fractional order complex chaotic systems, J. Comput. Nonlinear Dynam., 12 (2016), 8 pages. 1

[34] X. Song, S. Song, B. Li, Adaptive synchronization of two time-delayed fractional-order chaotic systems with different structure and different order, Optik, 127 (2016), 11860-11870. 1

[35] X. Song, S. Song, B. Li, I. T. Balsera, Adaptive projective synchronization for time-delayed fractional-order neural networks with uncertain parameters and its application in secure communications, Trans. Inst. Measurement Control, 40 (2017), 3078-3087. 1

[36] M. Srivastava, S. P. Ansari, S. K. Agrawal, S. Das, A. Y. T. Leung, Anti-synchronization between identical and nonidentical fractional-order chaotic systems using active control method, Nonlinear Dynam., 76 (2014), 905-914. 5

[37] J. W. Sun, Q. Yin, Y. Shen, Compound synchronization for four chaotic systems of integer order and fractional order, EPL (Europhys. Lett.), 106 (2012), 9 pages. 1

[38] Z. Wang, X. Huang, H. Shen, Control of an uncertain fractional order economic system via adaptive sliding mode, Neurocomputing, 83 (2012), 83-88. 1

[39] S. Wang, Y. Yu, M. Diao, Hybrid projective synchronization of chaotic fractional order systems with different dimensions, Phys. A, 389 (2010), 4981-4988. 1 
[40] G.-C. Wu, D. Baleanu, Chaos synchronization of the discrete fractional logistic map, Signal Process., 102 (2014), 96-99. 1

[41] X. J. Wu, H. T. Lu, S. L. Shen, Synchronization of a new fractional-order hyperchaotic system, Phys. Lett. A, 373 (2009), 2329-2337. 1

[42] Y. Xu, H. Wang, D. Liu, H. Huang, Sliding mode control of a class of fractional chaotic systems in the presence of parameter perturbations, J. Vib. Control, 21 (2015), 435-448. 1

[43] P. Zhou, R. J. Bai, The adaptive synchronization of fractional-order chaotic system with fractional-order $1<\mathrm{q}<2$ via linear parameter update law, Nonlinear Dynam., 80 (2015), 753-765. 1

[44] H. Zhu, S. B. Zhou, Z. S. He, Chaos synchronization of the fractional-order Chen's system, Chaos Solitons Fractals, 41 (2009), 2733-2740. 4

[45] M. Zribi, N. Smaoui, H. Salim, Synchronization of the unified chaotic systems using a sliding mode controller, Chaos Solitons Fractals, 42 (2009), 3197-3209. 1 